\title{
DESIGN AND IMPLEMENTATION OF A SENSORLESS SWITCHED RELUCTANCE DRIVE SYSTEM
}

\author{
**Bin-Yen Ma, *Tian-Hua Liu, *Ching-Guo Chen, *Tsen-Jui Shen, and ${ }^{* *} \mathrm{Wu}-$ Shiung Feng \\ *Department of Electrical Engineering \\ National Taiwan Institute of Technology \\ 43, Keelung Road, Section 4 \\ Taipei, Taiwan 106, R. O. C. \\ **Department of Electrical Engineering \\ National Taiwan University \\ 1, Roosevelt Road, Section 4 \\ Taipei, Taiwan 106, R. O. C.
}

\begin{abstract}
This paper presents a new sensorless switched reluctance drive system. By suitably shaping an induced voltage in an inactive phase which is adjacent to an energized phase of a switched reluctance motor, the shaft position of the rotor can be easily obtained. First, the theoretical analysis of the proposed method is presented. By systematic theoretical analysis, a voltage signal which can easily estimate the shaft position of the motor is derived. This signal is only related to the input dc voltage of the converter, and the self and mutual inductances of the motor. After that, the design for a simple circuit which can synthesize the required voltage signal for rotor position estimation is presented. Next, how a 32-bit microprocessor system is used to execute the position and speed estimation, speed-loop control, and current-commands generation is shown. Several experimental results validate the theoretical analysis. This paper presents a new direction in the design and implementation of a sensorless switched reluctance drive system.
\end{abstract}

This research is supported by the National Science Council, R. O. C. under grant NSC 86-2213-E011-076

\section{INTRODUCTION}

Switched reluctance motor (SRM) drive technology has been developed over the last two decades. The SRM system has many advantages. For example, both the motor configuration and the power converter are simple and rugged. There is no winding in the rotor. As a result, the SRM has a higher efficiency than the induction motor [1]. The sensorless drive is a new direction in the design and implementation of the SRM drive system. Papers on several techniques in this field have been published. For example, Lang et al. proposed state observers to determine the rotor position of the SRM. These observers, however, were only validated by computer simulations [2]. Another way to obtain the position information is to detect the phase current waveform and determine the position by calculating the phase inductance value from the changing rate of the phase current [3]. This method, however, requires injection of a diagnostic pulse to an inactive phase. This diagnostic pulse generates negative torque and reduces the performance of the drive system. Some researchers have used different approaches to estimate the rotor position of the SRM. Ehsani et al. proposed a linear inductance to 
time period converter in order to estimate the shaft position [4]. Though this approach is good and direct, a modulated circuit is required. The modulated circuit is difficult to realize and the signal is easily polluted by the pulse-widthmodulated (PWM) switching voltage as well. Recently, a sensorless method has been proposed by measuring a mutually induced voltage of an inactive phase of the SRM [5]. This is an effective way to estimate the shaft position of the SRM because it provides a clear relationship between the induced voltage and the rotor position. This method, unfortunately, has some disadvantages also. First, this method requires the design of a sample and hold circuit which is controlled by a synchronizing signal. The signal has to be synchronized with the PWM signals of the converter to detect whether the energized winding is operated in a conduction mode or in a free-wheeling mode. The circuit, therefore, is difficult to implement. Second, after the sample and hold circuit, the mutually induced voltage is related to the current command, the stator resistance, the mutual inductance, the selfinductance, and the rotor speed of the switched reluctance motor. The proposed sensorless method in paper [5], therefore, has some limitations. For example, at a low speed control range, a threedimensional table is required even under the assumption that the speed dependent terms are negligible. In addition, the proposed method can not be applied in a high speed range because the speed dependent terms enlarge the estimated position error. In this paper, we, therefore, propose a new method for solving the above mentioned problems.

\section{SYSTEM DESCRIPTION}

Fig. 1 shows the proposed closed-loop control diagram of the whole SRM drive system. The system consists of two major parts: a microprocessor system and some hardware circuits. The microprocessor system consists of a 32 bit CPU ( MC68020), a coprocessor ( MC68881), D/A converters, and A/D converters. The coprocessor can efficiently compute $\sin , \cos$, and floating point operations. Moreover, the MC68881 executes its computation concurrently with the MC68020 to reduce the required computation time of the whole system. The sampling interval of the microprocessor system, therefore, is only $0.4 \mathrm{~ms}$. The hardware circuits consist of some Hall-effect current sensors, a rectifying circuit, a shaping circuit, a current regulated converter, and a threephase, $10 \mathrm{hp}, 12 / 8 \mathrm{SRM}$. In the closed-loop drive system, first, the microprocessor reads the shaped mutual induced signal $v_{m o}$ of each phase via the A/D converter, and estimates the shaft position and speed of the SRM. Second, the microprocessor calculates the speed difference between the speed command and the estimated speed. Third, the microprocessor executes the speed-loop controller to determine the torque command $T_{e}{ }^{*}$. Next, the microprocessor maps the torque command to the current command amplitude $I^{*}$. After that, the microprocessor determines the three-phase current commands $I_{a}{ }^{*}, I_{b}{ }^{*}$, and $I_{c}{ }^{*}$ according to an estimated commutation angle $\theta_{0}$. The three-phase current commands are square wave with $1 / 3$ duty cycle. Finally, the microprocessor outputs the three-phase current commands to a current- 
regulated converter via the $\mathrm{D} / \mathrm{A}$ converters. The current-regulated power converter regulates the three-phase real currents to follow the current commands. The torque of the SRM, therefore, is generated to rotate the SRM. A closed-loop sensorless drive system is thus achieved. The system is flexible because the controller, the three-phase current command generator, and the estimator are executed by the microprocessor system. In addition, the system is suitable for application in industry because it is easily implemented.

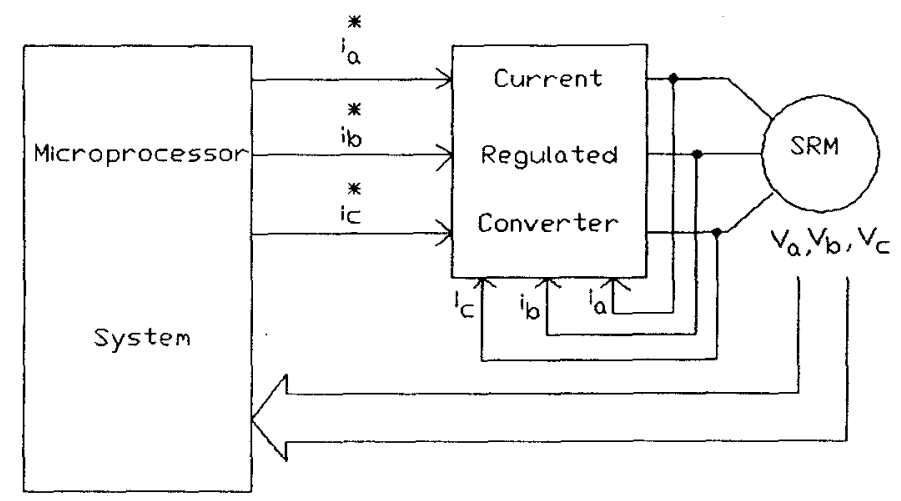

Fig. 1 Block diagram of the whole system.

\section{SENSORLESS TECHNIQUE}

In this paper, a circuit to detect the mutual induced voltage of the stator winding, which is inactive and in front of the currently energized winding, is designed. For example, if the b-phase is energized, the mutual induced voltage of the aphase is detected to determine the commutation angle (time) of turning off the b-phase as well as turning on the c-phase. This principle can be easily applied while the c-phase or the a-phase is energized. The systematic theoretical analysis is described as follows. The induced voltage of the mutual inductance for a SRM can be expressed as

$$
\begin{aligned}
& v_{m}=d \lambda_{m} / d t \\
& \lambda_{m}=L_{m} i
\end{aligned}
$$

where $v_{m}$ is the induced voltage, $\lambda_{m}$ is the mutual flux-linkage, $L_{m}$ is the mutual inductance, and $i$ is the energized current. Substituting (2) into (1),we can obtain

$$
v_{m}=L_{m} d i / d t+\omega_{e} i d L_{m} / d \theta_{e}
$$

where $\omega_{e}$ is the electrical rotor velocity of the motor. The energized voltage can be expressed as

$$
\begin{aligned}
v_{p h} & =i r+d \lambda_{p h} / \mathrm{dt} \\
& =i r+L_{s} d i / d t+\omega_{e} i d L_{s} / d \theta_{e}
\end{aligned}
$$

where $v_{p h}$ is the energized phase voltage, $r$ is the stator resistance, $\lambda_{p h}$ is the self-induced flux, and $L_{s}$ is the self-inductance. According to equation (4), we can easily obtain

$$
d i / d t=\left(1 / L_{s}\right)\left(v_{p h}-i r-\omega_{e} i d L_{s} / d \theta_{e}\right)
$$

After substituting equation (5) into equation (3), we can obtain

$$
\begin{gathered}
v_{m}=\left(L_{m} / L_{s}\right)\left(v_{p h}-i r-\omega_{e} i d L_{s} / d \theta_{e}\right) \\
+\omega_{e} i d L_{m} / d \theta_{e}
\end{gathered}
$$

The induced voltage is related to the switching mode of the energized winding. Fig. 2 shows the configuration of one leg of the converter. Then, we 
discuss the operation of the adjacent energized phase in Fig. 2. While the power devices $T_{1}$ and $T_{2}$ are conducted, the stator winding is operated in a conduction mode. In this mode, the phase voltage $v_{p h}$ of the measured winding is equal to the input dc supply voltage $V_{d c}$. The induced voltage, therefore, can be expressed as

$$
\begin{gathered}
v_{m c}=\left(L_{m} / L_{s}\right)\left(V_{d c}-i r-\omega_{e} i d L_{s} / d \theta_{e}\right) \\
+\omega_{e} i d L_{m} / d \theta_{e}
\end{gathered}
$$

where $v_{m c}$ is the mutual induced voltage while the adjacent phase is operated in a conduction mode. On the other hand, while the adjacent energized phase is operated in a free-wheeling mode, its power devices $T_{1}$ and $T_{2}$ are turned off. Then the diodes $D_{1}$ and $D_{2}$ are turned on immediately to maintain the current of the winding as a constant. Therefore, the phase voltage $v_{p h}$ is equal to $-V_{d c}$. The induced voltage can be expressed as

$$
\begin{gathered}
v_{m f}=\left(L_{m} / L_{s}\right)\left(-V_{d c}-i r-\omega_{e} i d L_{s} / d \theta_{e}\right) \\
+\omega_{e} i d L_{m} / d \theta_{e}
\end{gathered}
$$

where $v_{m f}$ is the mutual induced voltage while the adjacent energized phase is operated in a freewheeling mode. From equation (8), it is easy to understand that the voltage $v_{m f}$ is always negative because the last term is small. As a result, we can design a rectifier circuit to change the polarity of the voltage $v_{m f}$. After the process of the rectifier circuit, the induced voltage becomes a positive value, and is expressed as

$$
\begin{gathered}
v_{m r}=\left(L_{m} / L_{s}\right)\left(V_{d c}+i r+\omega_{e} i d L_{s} / d \theta_{e}\right)- \\
\omega_{e} i d L_{m} / d \theta_{e}
\end{gathered}
$$

where $v_{m r}$ is the signal of the rectified mutual induced voltage while its adjacent phase is operated at a free-wheeling mode. Observing the equations (7) and (9), the last three terms can be canceled by using a smoothing or averaging circuit. Many different circuits can provide this function. In this paper, a passive filter is designed to eliminate the high frequency harmonics and to smooth (average) the $v_{m c}$ and $v_{m r}$. The voltage at the output of the filter can be expressed approximately as

$$
\begin{aligned}
& v_{m o}=(1 / 2)\left(v_{m c}+v_{m r}\right) \\
& =\left(L_{m} / L_{s}\right) V_{d c}
\end{aligned}
$$

where $v_{m o}$ is the output voltage of the filter. The equation (10) is very attractive because the induced voltage at the output of the filter is only related to the input dc voltage of the converter, and the self and mutual inductances of the SRM. In addition, these inductances are related to the rotor position of the SRM. The rotor position of the SRM, therefore, can be easily estimated by detecting the output voltage of the filter.

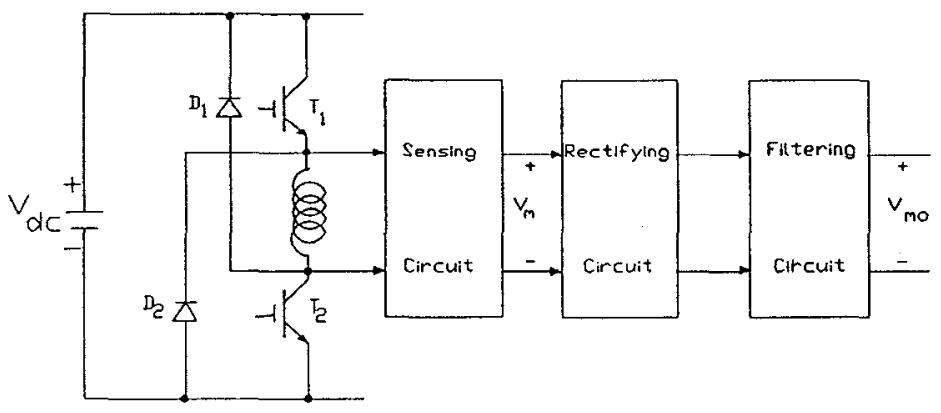

Fig. 2 The measuring and shaping circuit of the induced voltage. 


\section{EXPERIMENTAL RESULTS}

Some experimental results are shown here. While we executing the experiment, the SRM is coupled with a dynamometer, TM-5MT (DS), which is a product of the MEIWA company. The experimental waveforms are measured by a Tektronix digital storage oscilloscope-TDS 410. The parameters of the speed control are $K_{p}=3$ and $K_{I}=0.01$. The input voltage of the converter is $V_{d c}=$ $150 \mathrm{~V}$. The inertia of the motor and dynamometer is $0.025 \mathrm{Nt}-\mathrm{m}-\mathrm{sec}^{2} / \mathrm{rad}$. The hysteresis band is set at \pm 0.3 A. Some simulated and experimental results are shown here. Fig. 3(a) shows the calculated self-inductance of the SRM . Fig. 3(b) shows the measured mutual inductance which is obtained by exciting a 2 A constant current and then computing the mutual inductance. Fig. $4(a)$ is the measured a-phase voltage waveform that is detected at the output of the isolation amplifier circuit of the aphase. The measured waveform is switched between positive and negative because each leg of the power converter is operated between the conduction mode and the free-wheeling mode. From 0 to 30 degrees, the a-phase is energized by the PWM input voltage. However, due to the falling time of the current, the energized voltage is maintained continuously from 0 to 50 degrees. Beyond 50 degrees, the a-phase is blocked and the b-phase is energized. After that, the measured voltage is not an energized PWM voltage but is the induced voltage of the a-phase while the b-phase is excited. Fig. 4(b) shows the waveform that has been processed by the rectifying and filtering circuits. In this figure, the waveform is a smoothing wave. Beyond 50 degrees, the signal is an induced voltage which is related to the rotor position. The setting voltage level and the signal are crossed at 150 degrees. At this point, the bphase is blocked, and the c-phase is energized. As a result, we detect the a-phase induced voltage to determine the commutation time of turning off the b-phase and turning on the c-phase. Fig. 5 shows the rotor angles of the estimated angle and the real angle, which is detected by using an encoder. Fig. 6(a) shows the measured speed response of a 500 $\mathrm{r} / \mathrm{min}$ step input command. Fig. 6(b) shows the load disturbance rejection response of the system at 500 $\mathrm{r} / \mathrm{min}$ and $2 \mathrm{Nt}-\mathrm{m}$. Fig. 7 shows the correspondent (500 r/min, 2Nt-m) steady-state a-phase current and a-phase voltage. Fig. 8 shows the speed response of the system at a $50 \mathrm{r} / \mathrm{min}$ step input command.

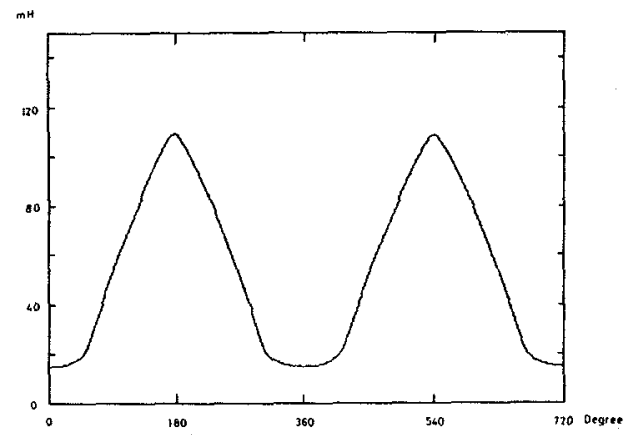

(a)

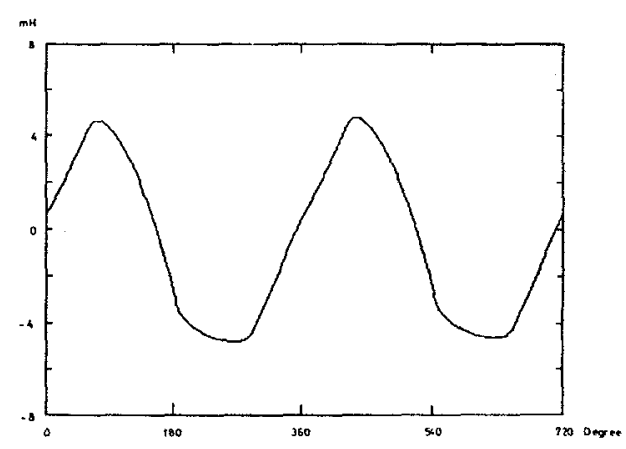

Fig. 3 The measured inductances (a) self (b) mutual. 


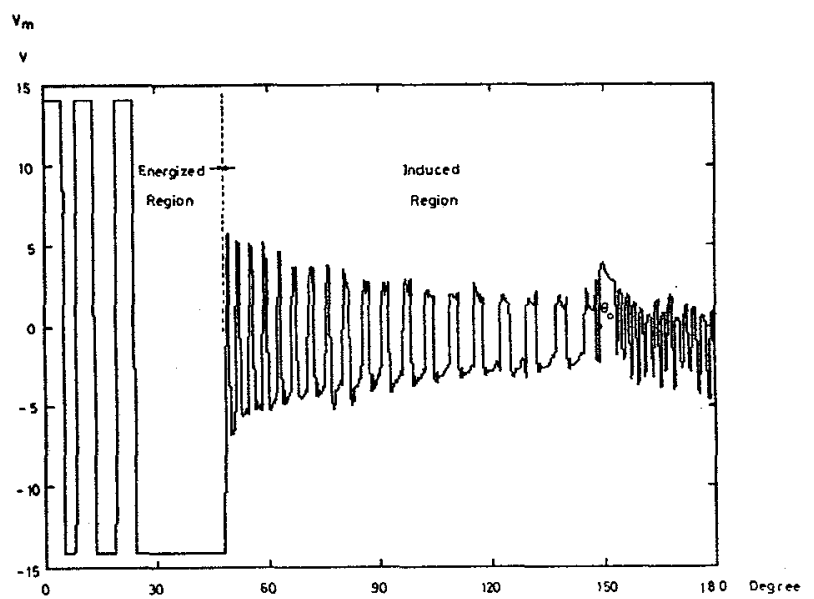

(a)

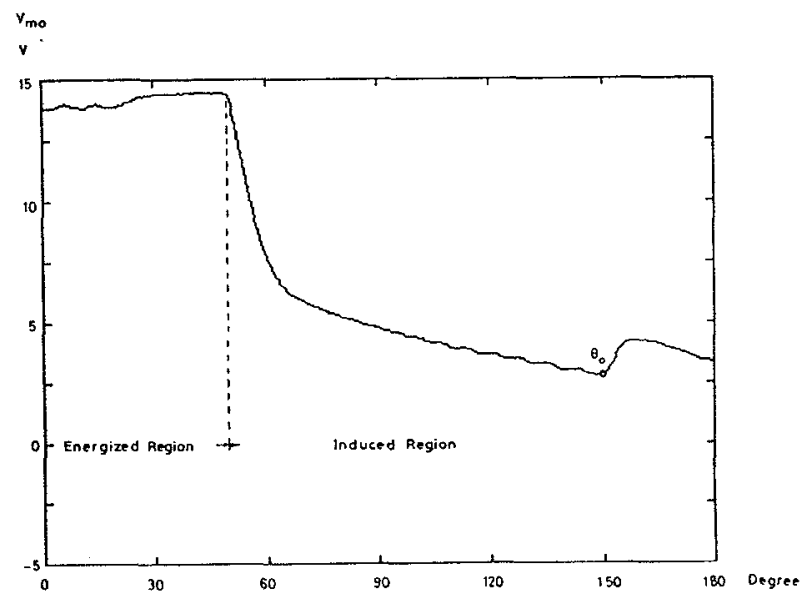

(b)

Fig. 4 The measured induced voltage

(a) before rectifying (b) after filtering.

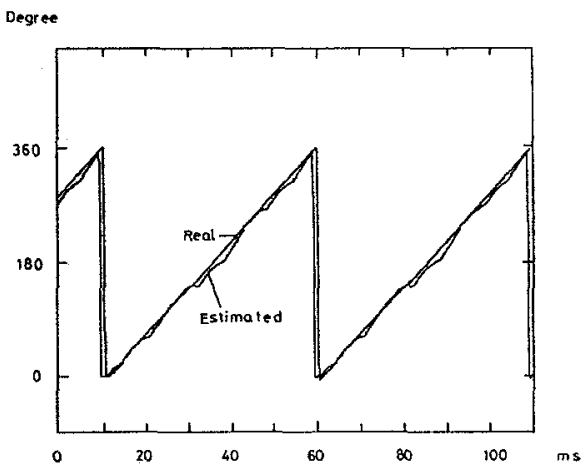

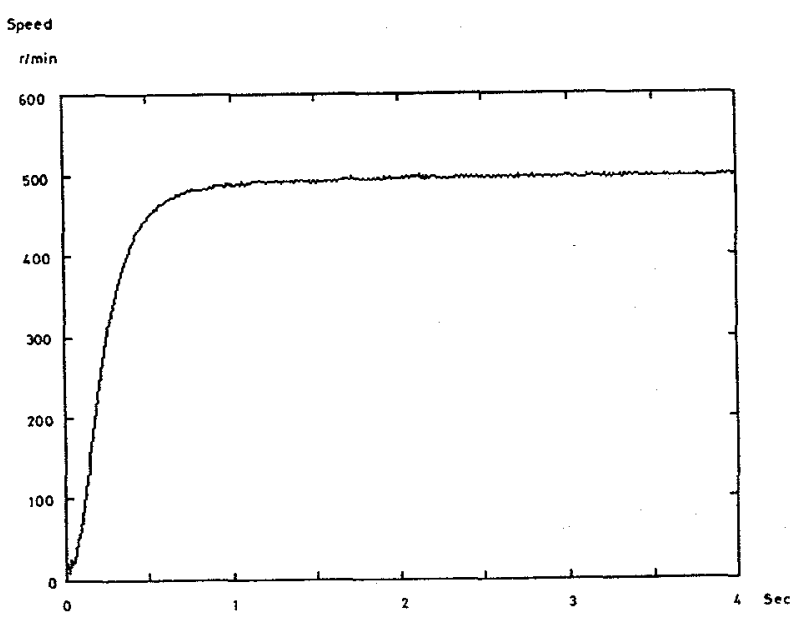

(a)

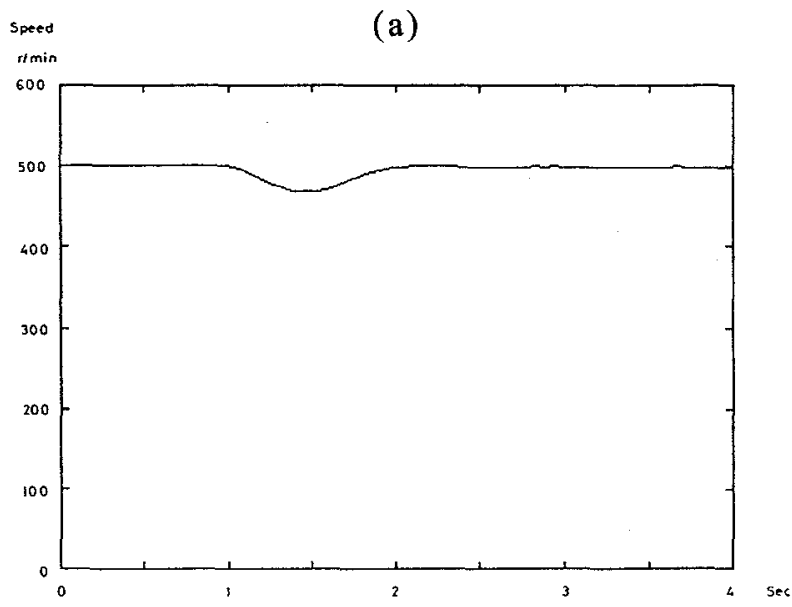

(b)

Fig. 6 The measured speed response at $500 \mathrm{r} / \mathrm{min}$ . (a) transient (b) load disturbance.

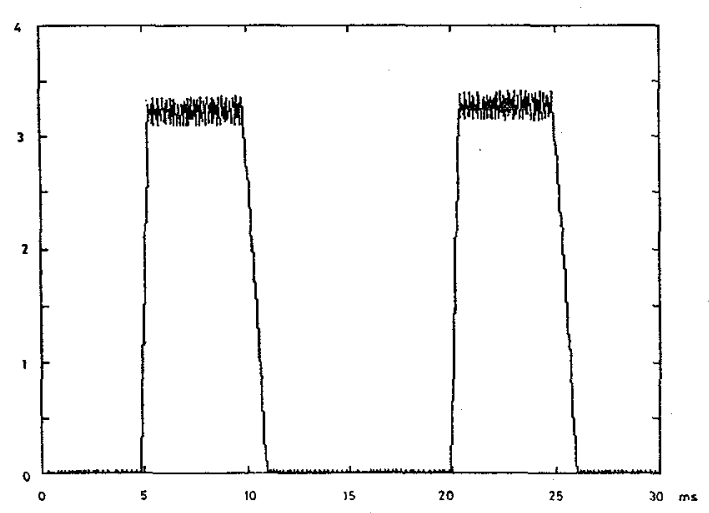

(a)

Fig. 5 The estimated and real rotor angle. 


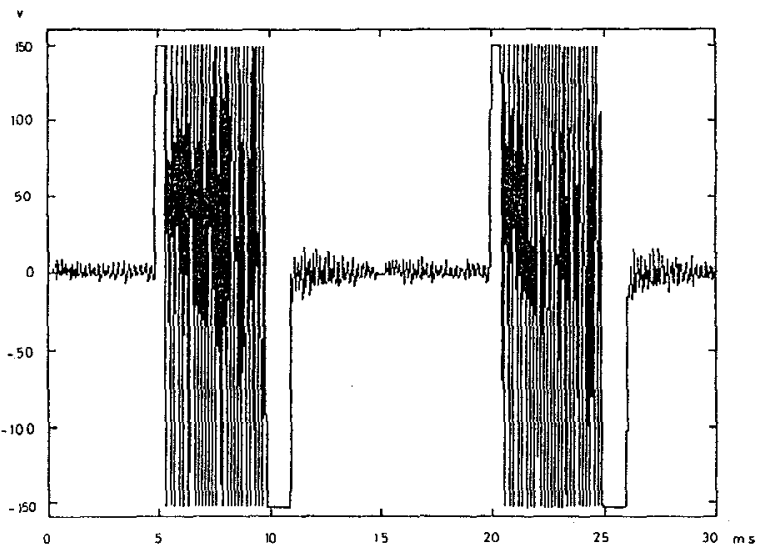

(b)

Fig. 7 The measured steady-state waveforms

(a) a-phase current (b) a-phase voltage.

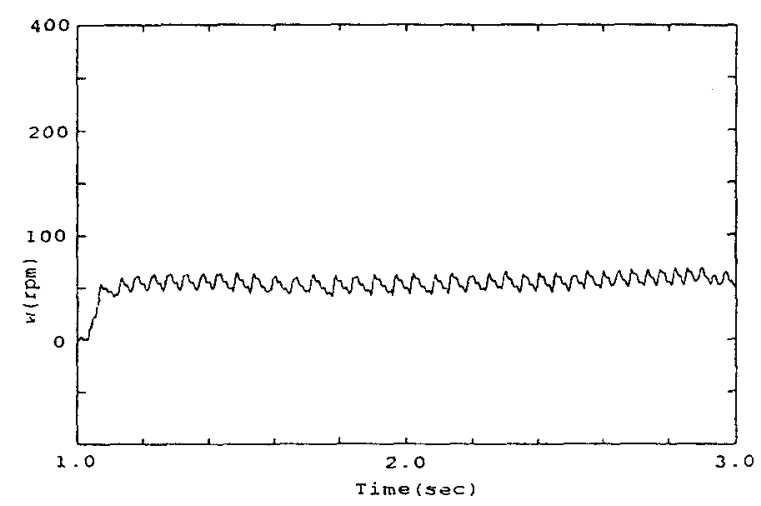

Fig. 8 The measured speed response of the sensorless drive at $50 \mathrm{r} / \mathrm{min}$.

\section{CONCLUSIONS}

In this paper, a simple method of designing and implementing a sensorless switched reluctance drive system has been presented. By designing a simple circuit, the commutation angle of the SRM has been easily determined. Then, a microprocessor based sensorless SRM drive has been implemented. The sensorless drive system performs well. Several experimental results show the feasibility and correctness of the proposed sensorless method. The method proposed in this paper can easily be applied to actual industrial use due to its simplicity. This paper presents a new direction in the design of a sensorless switched reluctance drive system.

\section{REFERENCES}

[1] T. A. Lipo, "Recent progress in the development of solid-state ac motor drives," IEEE Trans. on Power Electron., vol. 3, no. 2, pp. 105-117, Apr. 1988.

[2] A. Lumsdaine and J. H. Lang, "State observers for variable-reluctance motors," IEEE Trans. on Ind. Electron., vol. 37, no. 2, pp. 133-142, Apr. 1990.

[3] W. D. Harris and J. H. Lang, "A simple motion estimator for variable- reluctance motors," IEEE Trans. on Ind. Appl., vol. 26, no. 2, pp. 237-243, Mar./Apr. 1990.

[4] M. Ehsani, I. Husain, and A. B. Kulkarni, "Elimination of discrete position sensor and current sensor in switched reluctance motor drives," IEEE Trans. on Ind. Appl., vol. 28, no. 1, pp. 128-135, Jan./Feb. 1992.

[5] I. Husain, M. Ehsani, "Rotor position sensing in switched reluctance motor drives by measuring mutually induces voltages," IEEE Trans. on Ind. Appl., vol. 30, no. 3, pp. 665-672, May/June 1994. 